\title{
An Analysis of Time-delay for Remote Piloted Vehicle
}

\author{
Feng Wang, Shengjun $\mathrm{Qi}^{\mathrm{a}}$ and Li Jing \\ Chinese Flight Test Establishment, 710089 Xi'an, China
}

\begin{abstract}
Time-delay is one key factor affecting handling qualities of remote piloted vehicle. In this paper, the composition elements of time-delay were analyzed, in order to determine the relationship between latency and handling qualities. Then, comparing with the definition of time-delay in manned aircraft operation, the expression of time-delay was proposed for remote piloted vehicle and the measuring method of time-delay in piloted airplane was used as a reference. Moreover, considering different types of time-delay in RPV operation, typical openloop and closed-loop experiments were designed and conducted based on ground testing environment. According to these results, the relation between time-delay and handling qualities was obtained. Finally, validity of this relationship was demonstrated through simulated approach and landing during flight testing. Time-delay is a critical element of RPV handling qualities, the research of which will be helpful and meaningful in unmanned aerial vehicle design and flight testing.
\end{abstract}

\section{Introduction}

For remote piloted unmanned aerial vehicle, pilot in ground control station could not get real time feedback as their counterpart, manned aircraft pilot, such as status of aircraft, visual weather and vibration of fuselage. Consequently, vehicle could not receive real time control order via uplink from ground control station. Therefore, time-delay in unlink and downlink has been becoming one primary issue affecting operator who control UAV to complete assigned mission. Nowadays, a large amount of research are carried out to decrease influence of time-delay on handling qualities in remote piloted vehicle and many methods are applied on RPV operation to alleviate operator's workload and to improve handling qualities. However, there are still two unsolved questions: how to define time-delay in unmanned aerial vehicle clearly and how to determine relation between time-delay and handling qualities. In this paper, study both in theoretical analysis and flight testing is started to solve these two questions mentioned above.

\section{Definition of time-delay in remote piloted vehicle}

\subsection{Time-delay in manned aircraft}

In the standard of manned aircraft flying qualities, there are two kinds of time-delay definition: equivalent time-delay and effective time-delay [1-2]. Equivalent time-delay is mainly aiming at low

\footnotetext{
${ }^{a}$ Corresponding author:saintknight372@hotmail.com
} 
level equivalent system. The effective time-delay refers to the time that equals to number of the maximum rate of slope intersecting with time axis, which input of control force/displacement order produce.

Table 1 shows requirements in handling qualities of piloted aircraft. Time-delay in handling qualities of manned aircraft is measured by interval from input of con to response of control surface in either equivalent or effective time-delay.

Table 1. Time-delay requirement in handling qualities

\begin{tabular}{|c|c|c|}
\hline Scale & $\begin{array}{c}\text { Equivalent } \\
\text { time-delay } \\
(\mathrm{s})\end{array}$ & $\begin{array}{c}\text { Effective } \\
\text { Time-delay } \\
(\mathrm{s})\end{array}$ \\
\hline 1 & 0.1 & 0.12 \\
\hline 2 & 0.2 & 0.17 \\
\hline 3 & 0.25 & 0.21 \\
\hline
\end{tabular}

\subsection{Unmanned aerial vehicle time-delay based on handing qualities}

\subsubsection{Composition of UAV time-delay}

Time-delay of unmanned aircraft system consists of many items, not only the latency from input of manipulator system to response of control surface, but also latency of data link, digital processing in computers, and so on [3-5]. Comparing with latency of aircraft response, magnitude of data link timedelay is greater. Figure 1 is the composition diagram of time-delay in remote piloted vehicle. The total time delay is the sum of time-delay in ground control station, time delay of data link and time delay in UAV. The time delay on ground contains pilot input, computer calculation in ground control station and interaction between ground control station and data link, while time delay in UAV covers calculation in flight control computer, response of actuator and servo, interaction between aircraft and data link. And time delay of data link includes uplink latency and down link latency. It can be seen that the compositions of UAV time delay is rather complex. There are so many factors affecting the analysis of time delay of UAV.

\subsubsection{Definition of Unmanned Aerial Aircraft Time-delay}

Like definition of manned aircraft time-delay, time-delay of RPV reflects the relation in timedifference between input of order sent by pilot from GCS and display in screen of GCS down link from aircraft [6]. For the remote control, this expression reflexes pilot's feeling of latency in aircraft motion after manipulation, which has approximation for large time-delay RPV control as well as less accurate. It is difficult to measure equivalent time-delay and the main method for measurement is depends on equivalent matching algorithm to calculating latency. For the RPV with large magnitude of time-delay, adaptive condition happens easily. Hence, a pre-knowledge hypothesis is proposed for time-delay of data link.

Taking longitudinal movement of manned aircraft as an example, pilot got feedback of pitch angular acceleration firstly during manipulation, but it is difficult to measure acceleration in flight testing. However, as the integral of angular acceleration, angular velocity refers to variation of angular acceleration directly, and angular speed is easy to measure and has high accuracy. Therefore, that is why using angular speed as main parameter to evaluate time-delay. 
For remote piloted vehicle, pilot in GCS could not get feedback on change of angular speed, but get information about attitude instead. Hence, it is suggested to choose attitude as main evaluation indicator for time-delay. Meanwhile, from perspective of measurement and calculation, the most part of latency is led by data link, so it is not to emphasize precision of latency in aircraft. Considering all points mentioned above, the definition of time-delay in RPV is proposed as follows. The time-delay in RPV refers to time span from sending input of manipulating order by remote pilot in GCS to display of status of system in GCS via down linking after response of aircraft to input.

\subsubsection{Measurement of UAV time-delay}

There are two kinds of measurement for time-delay in manned aircraft. Equivalent time-delay is the equivalent matching of angular speed, while effective time-delay is calculated on basis of maximum slope of angular speed and starting time of motion. The time-delay of RPV is to measure time-relation between sending input of manipulation order and receiving status of aircraft shown in screen of GCS. Thus, variation of attitude angle is becoming the main parameter in time-delay measurement. Operator in GCS could monitor changing speed of RPV attitude. The method of equivalent time-delay in manned aircraft could be used in RPV time-delay measurement to determine slope of attitude angle. As shown in figure 1, Step input starts in first second. The time-delay of manned aircraft is the difference value between starting time of order and crossover point of maximum slope and time axis, which is $\Delta \mathrm{t}$. As shown in figure 1, according to measurement of manned aircraft, time-delay of RPV is the interval between start time of step input and the time, which value equals crossover point of maximum slope on attitude angle and time axis.
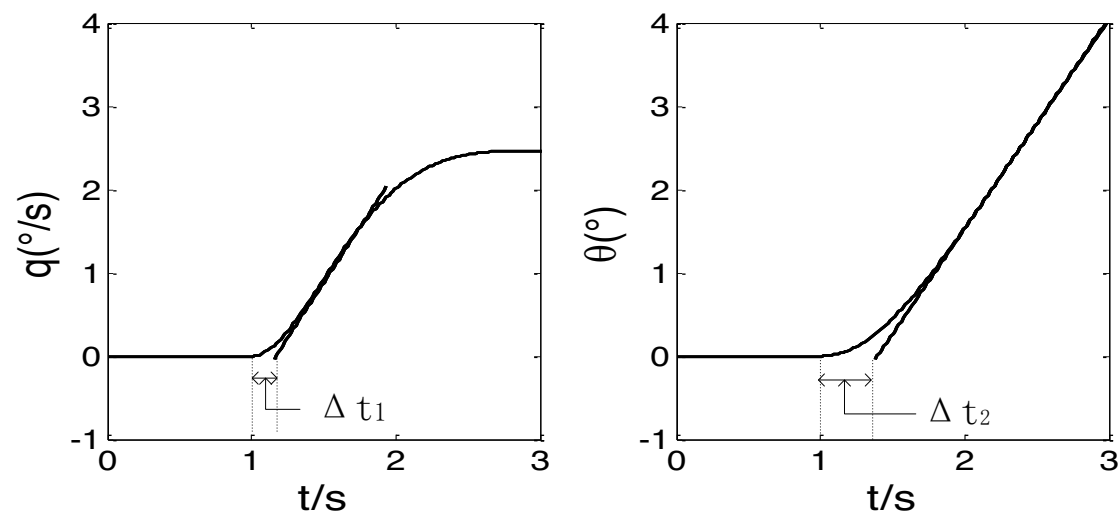

Figure 1. The method of measuring time-delay

\section{Testing environment for time-delay}

Closed-loop ground test and flight test are conducted in small unmanned aerial aircraft in order to verify the relationship between time-delay and handling qualities.

\subsection{Closed-loop ground testing introduction}

Closed-loop ground testing system is built using simulator and aircraft, which is shown in figure 2 . Firstly, input of manipulation input was sent to dual-redundancy flight control computer through data link. Then, Flight control computer will send orders to actuator to deflect control surface. On the other side, analog computer collects deflection of surface and calculates dynamic response of aircraft, which 
will be transmitted to ground control station via flight control computer and data link. All of process forms a closed-loop.

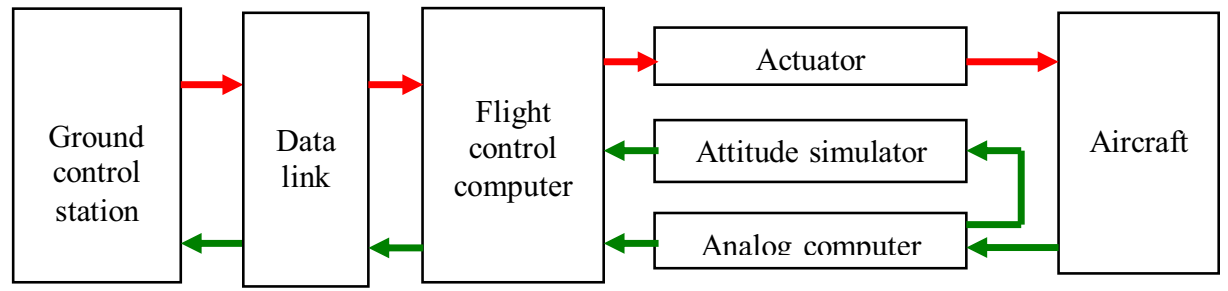

Figure 2. Closed-loop ground test composition

\subsection{Flight testing description}

The experiment is executed in one remote piloted aircraft that was refitted from trainer aircraft. In order to achieve remote control, fly-by-wire system was added on the basis of mechanical manipulation system in front of cockpit, so that pilot in front could control vehicle through fly-bywire. The pilot in rear of cockpit still uses mechanical manipulation system as safe guarantee. In the ground control station, side stick was installed and layout of human-machine interface was optimized to make sure operation safety and effectiveness. On the other hand, data transmission system with high-performance was employed in order to decrease latency in data link, which means input of order and status of system could interact in short interval.

During testing, remote pilot control the RPV in GCS to measure the period from sending input of control to receiving status on screen.

\section{Results analysis}

\subsection{Analysis of closed-loop test results}

There are 5 sophisticated test pilots having more than 3000 flight hours in fixed wing manned-aircraft, who participate in testing. In the beginning phase of testing, pilots did not adapt to large time-delay manipulation. They can complete open-loop mission with easy input like impulse and step, while it is hard to accomplish closed-loop task using simple input. With much practice, pilots understand the characteristics of RPV time-delay and influence on aircraft motion from different magnitude of latency, and they could finish tracking task in closed-loop test.

The comments from pilots are shown in table 2. The open-loop task includes impulse and step input maneuvers, while closed-loop mission refers to approaching with inspecting disturbance and correcting. Since time-delay is measured on the basis of attitude, the magnitude of time-delay in RPV is greater than manned aircraft. From comments, it is seen that well-trained pilot could accomplish remote control in open-loop task and there is higher probability of successful landing with simulated correcting ability when magnitude of time-delay is around 300 milliseconds. When magnitude locates in 410-460 milliseconds, the operative difficulty becomes greater and coupling oscillation occurs when correcting during landing. Over 510 milliseconds, it is hard to execute normal landing, but there is less probability of occurring coupling oscillation.

With analysis of testing data, time-delay for 400-500 milliseconds is a sensitive range. The magnitude of time-delay coincides with responding time, which may produce coupling phenomenon.

Table 2. Comments about closed-loop test from pilots

\begin{tabular}{|l|l|l|}
\hline No. & Time-delay(ms) & Closed-loop operation \\
\hline
\end{tabular}




\begin{tabular}{|c|c|c|}
\hline 1 & 310 & Task can be accomplished \\
\hline 2 & 360 & $\begin{array}{c}\text { Task can be accomplished, but need more mental } \\
\text { effort }\end{array}$ \\
\hline 3 & 410 & $\begin{array}{c}\text { Hard to accomplish precise landing under large } \\
\text { Disturbance, more probability for oscillation }\end{array}$ \\
\hline 4 & 460 & $\begin{array}{c}\text { Disturbance causing Coupling oscillation, } \\
\text { difficult accomplish to landing }\end{array}$ \\
\hline 6 & 510 & $\begin{array}{c}\text { Coupling oscillation, pilot cannot accomplish } \\
\text { normal landing。 }\end{array}$ \\
\hline 7 & 560 & Hard to accomplish precise heading \\
\hline
\end{tabular}

\subsection{Analysis of flight test results}

In order to explore further relationship between time-delay and handling qualities, remote pilot control vehicle carried out simulated approach at $80 \mathrm{~m}$ height, and then go around, shown in figure 3 . During testing, remote pilot adjust heading to targeting at runway. It is found that heading is difficult for remote pilot. And it is hard to touchdown. Therefore, Go-around is the only choice for remote pilot due to safety consideration.

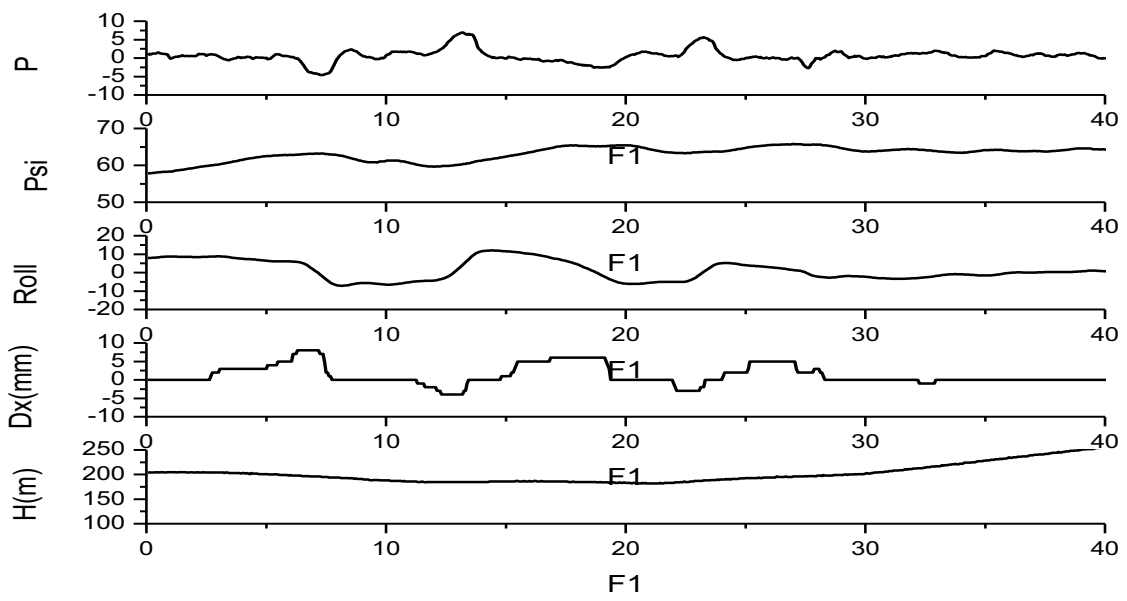

Figure 3. Simulated approach and go around in remote piloted control mode

\subsection{Comparison between tests}

During approaching phase, step input of heading operation was repeated for several times to determine magnitude of time-delay. The results illustrate that time-delay in heading direction is around 400 milliseconds, as shown in figure 4. This magnitude of latency is almost the same with 
comments from remote pilots. Hence, the scope of remote operation time-delay locates between 400 500 milliseconds, and manipulation, especially closed-loop operation is hard to implement in this range, which would cause piloted induced oscillation .

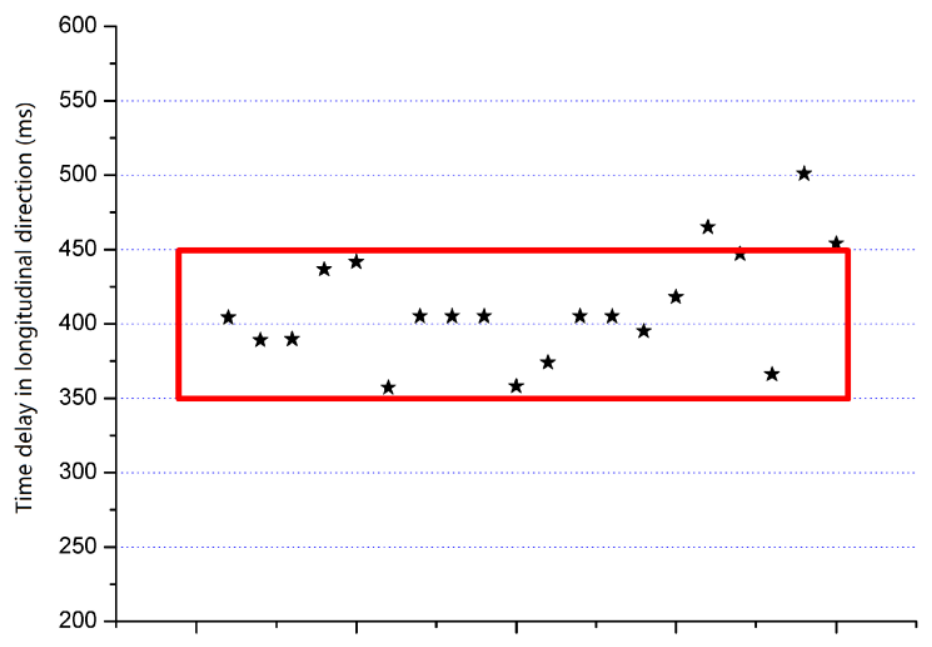

Figure 4. Statistics of heading time-delay

Finally, it is seen that the results between closed-loop ground test and flight test coincide badly based on analysis. From definition and results mentioned above, it is hard to control remote piloted vehicle if magnitude of time-delay is greater than 400 milliseconds.

\section{Conclusions}

As development of UAV and expanding in applications, latency in operation has been becoming one of primary issues in operation. From perspective of handling qualities, definition and composition of remote piloted vehicle are presented at first. To demonstrate relationship between time-delay and handling qualities, closed-loop ground test and flight test are executed. Results show that scope of latency is found and suggestions are proposed, which is helpful for study of remote piloted vehicle handling qualities in future.

\section{References}

1. Department of Defense,MIL-HDBK-1797, 20(1997)

2. C.Mcfalan, T.Richardson, C.Jones, AIAA,7156(2008)

3. T.Yu Jin, W. Jian Pei. F 28,13(2010)

4. W.Fang, F.Wang,T.Ding Jie. F 28, 68 (2010)

5. T.Ding Jie, W.Fang, F.Wang. F 29, 17 (2011)

6. S.C.de Vries. UAVs and Control delays.15 (US,2005) 